\title{
Female sterilisation: is it what women really want?
}

\author{
Alison Mattinson, MRCGP, MFFP, SCMO; Diana Mansour, FRCOG, MFF, Consultant in Community Gynaecology and \\ Reproductive Health Care, Family Planning Unit, Royal Victoria Infirmary, Newcastle-upon-Tyne, UK
}

Correspondence: $\operatorname{Dr}$ A B Mattinson, Family Planning Unit, Victoria Outpatient Department, Royal Victoria Infirmary, Queen Victoria Road, Newcastle-upon-Tyne NE1 4LP, UK

(Accepted 25th January 2003)

Journal of Family Planning and Reproductive Health Care 2003; 29(3): 136-139

\begin{abstract}
Objective. To audit the effectiveness of a female sterilisation counselling clinic.

Design. A prospective, observational study of a female sterilisation counselling clinic over a 6-month period to determine the proportions of attenders keeping their appointment, proceeding to sterilisation, and attending for surgery.

Setting. A hospital-based, city centre family planning clinic run by permanent, family planning-trained staff.

Results. A total of 226 women had appointments for female sterilisation counselling and most confirmed attendance. Only 153 (68\%) women attended; of those 101 (66\%) chose to be sterilised, the remainder choosing reversible contraception. Ninety-three (92\%) women went ahead with the sterilisation procedure. Thus only $61 \%$ of the original attenders were sterilised; this was only $41 \%$ of those referred.

Conclusions. The study suggests that many women referred for sterilisation counselling fail to attend. This finding needs to be further explored. For those women who do attend, accurate information and informed counselling is important. Many women are still unaware of highly effective long-term reversible contraception, and following discussion decide against female sterilisation. Services offering counselling for female sterilisation should also be able to provide easy access to a full range of contraceptive methods.
\end{abstract}

\section{Key message points}

- A large number of women $(32 \%)$ failed to keep their appointment for sterilisation counselling.

- Many women are still unaware of highly effective, long-term, reversible methods of contraception.

- After sterilisation counselling $34 \%$ of women chose alternative forms of contraception.

- The number of female sterilisations performed in England and Wales is falling. Between 1998 and 2001 the number fell by $27 \%$. This may be due to the availability of other long-term reliable methods of contraception.

\section{Introduction}

Female sterilisation is the most widely used method of contraception worldwide. ${ }^{1}$ Although female sterilisation is considered a simple gynaecological procedure, it attracts high levels of litigation related to pre-procedure counselling, surgical complications and failures. ${ }^{2}$ Many gynaecologists are of the opinion that female sterilisation should take the back burner in view of the availability of alternative methods which are reversible, less invasive but just as effective. ${ }^{3}$

This prospective audit was embarked upon because of growing concern at the non-attendance for sterilisation counselling and also because many women seen were unaware of effective contraceptive alternatives to female sterilisation. We decided to evaluate, whether after informed counselling within a family planning service, women still wished to undergo female sterilisation or use alternative forms of contraception.

The Royal Victoria Infirmary (RVI) is a large teaching hospital in the centre of Newcastle-upon-Tyne. All female sterilisations in the Newcastle area are performed by the gynaecology department in this hospital. Until 1996 the counselling for female sterilisation was done in busy gynaecology outpatient clinics, often by junior medical staff who had very little family planning training. In 1996 it was decided that female sterilisation counselling would be better performed in the hospital family planning clinics (FPCs) where it could be undertaken in an unhurried atmosphere by staff trained in contraception and sexual health. These clinics are run by clinical medical officers and by family planning-trained nurses. A consultant gynaecologist takes overall responsibility for the clinics.

There are four general FPCs clinics at the hospital each week. On average, 10 women have appointments each week during these clinics for sterilisation counselling. The women are referred to the FPC by their general practitioners (GPs) and are sent a letter enclosing the time of their appointment and details of what to expect at their visit to the clinic.

They are also sent Family Planning Association leaflets about sterilisation and other contraceptive methods. ${ }^{4}$ The women are asked to confirm that they will be attending their appointment by telephoning the clinic secretary. Failure to do this within 3 weeks of the appointment date may result in the appointment being allocated to someone else. The average time from receiving the referral to the appointment date is 6 weeks.

Most women attend the sterilisation appointment on their own but some are accompanied by their husband, partner, friend or other family member. First they are seen by one of the nurses who takes a comprehensive history and performs blood pressure, height and weight measurements. We have produced a sterilisation counselling proforma using the Royal College of Obstetricians and Gynaecologists guidelines to ensure clear documentation. ${ }^{5}$ The nurse also discusses male and female sterilisation and alternative methods of contraception, especially long-acting, reversible methods such as the levonorgestrel intrauterine system (IUS) and progestogenonly implant (Implanon).

The women are then seen by the doctor for further counselling. Sterilisation has been a common source of litigation in gynaecology, accounting for $6 \%$ of claims notified to the Medical Defence Union. ${ }^{6}$ Therefore time is taken to discuss the permanence, irreversibility and failure rate of sterilisation (1:200 over a lifetime and up to onethird of which may be ectopic pregnancies). ${ }^{7}$ The operation techniques are discussed. At the RVI the majority of female sterilisations are performed laparoscopically using Filshie clips to occlude the Fallopian tubes. All women are 
informed, however, that a mini-laparotomy may be necessary should there be difficulties performing the laparoscopy or visualising the pelvic organs. This is particularly likely if the woman is obese, has had previous abdominal surgery or complications arise during the operation. The complications (haemorrhage, infection, bowel perforation), side effects and recovery time of the operation are discussed. Women are given a printed information sheet reminding them of points that have been discussed in the clinic.

Alternative methods of contraception are discussed with all women. Many women are still unaware of other highly effective, long-acting, reliable methods such as the IUS and contraceptive implant and their non-contraceptive advantages in relation to menstruation. There were no pregnancies in studies published concerning Implanon, suggesting it may be more effective than female sterilisation. $^{8}$ If a woman prefers to use one of these methods arrangements are made for IUS fitting or Implanon insertion. If the woman and her partner wish to know more about vasectomy they are referred to the weekly vasectomy counselling clinic located in the same unit and run by a dedicated medical practitioner.

Following counselling, if women still wish to be sterilised an abdominal and pelvic examination is carried out together with a cervical smear for cytology if appropriate. A blood sample is taken for a full blood count and a written consent to the procedure is obtained.

Current contraception is also discussed to ensure that women will be protected up until the time of surgery. Short-term contraception such as condoms, oral contraceptive pills or Depo-Provera can be given in the clinic.

Arrangements are then made for the women to be admitted to the hospital for laparoscopic sterilisation. Most women have the operation on the Day Unit and they are given their operation date before they leave the FPC. A few women are admitted to a gynaecology ward for their operation if it is felt there may be complications or they have other medical problems. Almost all women have had their operation within 3 months of attending the FPC for counselling.

\section{Method}

All women given appointments for female sterilisation counselling in the 6-month period January-June 2001 were included in the study. A data collection sheet was designed to record the woman's age, date of appointment and whether the appointment was kept or not. For women who attended the clinic details of number of children, marital status and current contraception were also recorded. Women were then counselled in the usual way as described in the Introduction.

After counselling, for those women who still wished to have a laparoscopic sterilisation arrangements were made for them to be admitted to the hospital for their operation. The reasons for wanting a sterilisation were recorded. It was recorded whether they were to be admitted to the Day Unit (no complications expected) or a gynaecology ward (possible complications or medical problems). The case notes were reviewed a few months later to confirm that the women had gone ahead with their operation.

Following counselling, some women decided not to have a sterilisation but to use alternative forms of contraception. The reason for this was noted on the data collection sheet. If the IUS or Implanon were chosen arrangements were made for insertion. It was later noted whether or not they attended for this procedure.

Some couples wished to be referred for vasectomy counselling. It was recorded subsequently whether they attended this appointment and whether a vasectomy operation was undertaken.

\section{Results}

Attendances for sterilisation counselling

In the 6-month period January-June 2001, 226 women were given appointments for sterilisation counselling having been referred to the FPC by their GPs. One hundred and fifty-three $(68 \%)$ women attended.

Ages of women referred for sterilisation counselling The ages of the women who attended for counselling ranged from 19 to 47 years with a median age of 33 years. The age range of the non-attenders was very similar with a median age of 32 years.

\section{Number of existing children}

The 153 women who attended their counselling appointment had between 0 and 7 children with a mean of 2.4. Women with no children were generally older (mean age 38 years) than those with 5, 6 and 7 children (mean ages 30,33 and 28 years, respectively).

\section{Marital status}

Less than half $(64 ; 43 \%)$ of the women requesting sterilisation were married, although many single women described their relationship as permanent.

\section{Contraceptive methods used at the time of counselling}

At the counselling appointment women were asked about the method of contraception they were currently using. A variety of contraception was being used (Figure 1). The most commonly used methods were combined oral contraceptives (COCs) used by $42(27 \%)$, condoms used by $35(23 \%)$ and the progestogen-only pill (POP) used by 25 $(16 \%)$ women. The women in this study did not wish to have more children and yet it is interesting to note that 15 (9\%) were using no contraception and seven $(5 \%)$ were using the withdrawal method.

Women choosing female sterilisation following counselling Following counselling, two-thirds of women $(101 ; 66 \%)$ chose female sterilisation and one-third $(52 ; 34 \%)$ chose alternative forms of contraception.

Of those 101 women choosing sterilisation, 78 (77\%) were suitable for day case surgery. Twenty-three $(23 \%)$ women did not fulfil the criteria for admission to the Day Unit and arrangements were made for them to be admitted to a gynaecology ward for their operation. The majority of these women were overweight (body mass index > 27) but a few had other medical problems such as a valvular heart disorder, a pacemaker, severe rheumatoid arthritis, two or more previous Caesarean sections or an alcohol and drug problem.

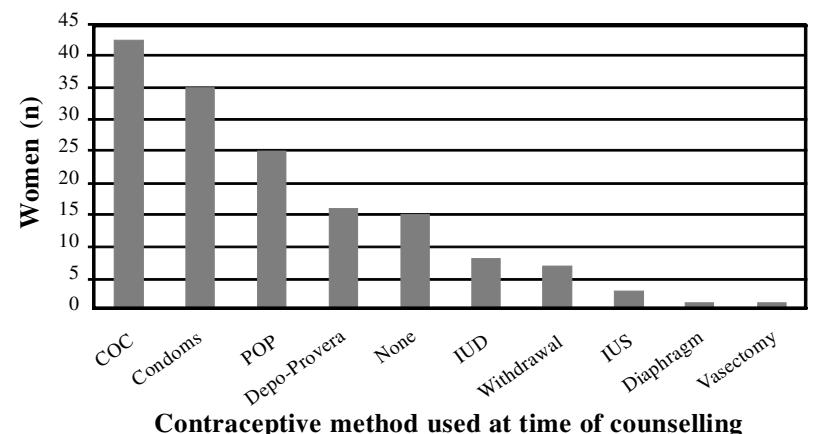

Figure 1 Contraceptive method used at the time of counselling $(n=153)$. COC, combined oral contraceptive; IUD, intrauterine device; IUS, intrauterine system; $P O P$, progestogen-only pill. 
Six $(6 \%)$ women cancelled or did not attend hospital for their operation. Two (2\%) women had their operation put 'on hold' because of other medical problems, which were being treated first. Therefore of the 101 women choosing female sterilisation only $93(92 \%)$ went ahead with the procedure. Of the 153 women who attended for sterilisation counselling $93(61 \%)$ went ahead with the operation. From the original 226 referral appointments only 93 (41\%) women had a female sterilisation procedure (Figure 2).

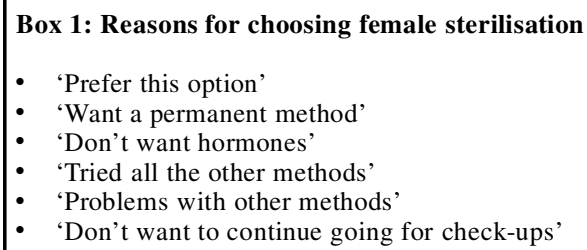

Women choosing alternative methods of contraception Following counselling, 52 (34\%) women chose a variety of alternative methods of contraception (Figure 3 ). The IUS was chosen by 19 (37\%) women and Implanon by 13 $(25 \%)$. Vasectomy was chosen by six couples. Four women were undecided after counselling. They were given literature to take away and read and appointments were made for them to return to the clinic to discuss things further. All four women failed to keep their follow-up appointments. Six women decided to continue with their contraceptive pills: three with COCs and three with the POP. Two women were referred to a gynaecologist for consideration of a hysterectomy because of severe menorrhagia. One of these women had been using the IUS for a year with no improvement of her menstruation. The other woman was also being treated for 'abnormal' smears. Both women underwent hysterectomies. One woman decided to continue with Depo-Provera following her counselling. One very young woman left the clinic before the consultation had finished. She was unhappy when she was told that in view of her young age she would need a second opinion about having a sterilisation. Alternative methods were unacceptable to her. She was sent an appointment to return to the clinic to see the consultant but failed to attend.

\section{Box 2: Reasons for choosing the IUS}

'Easy and avoids an operation'

'As effective as a tubal-tie'

'Will help with heavy periods as well as contraception

'Reversible - not in a stable relationship'

Women choosing the IUS

For women who decided not to have a female sterilisation the IUS was the most commonly chosen alternative.

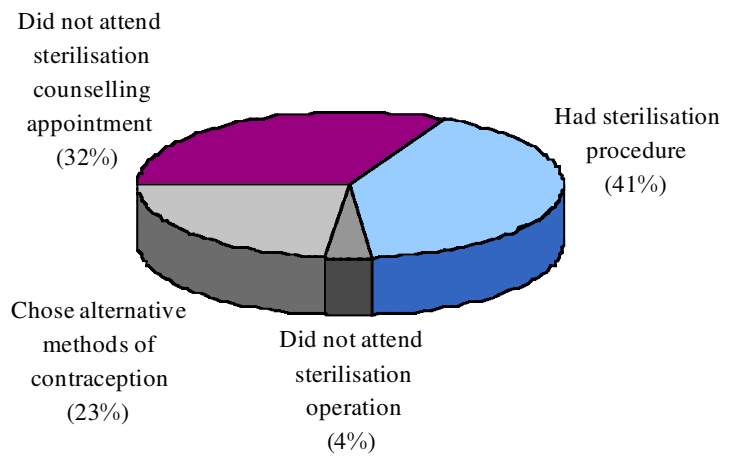

Figure 2 Outcome following female sterilisation referral
Nineteen women chose this method. They were examined, had chlamydia swabs taken and arrangements were made for them to attend the clinic to have the device inserted. Thirteen $(68 \%)$ women kept their appointment and had the IUS inserted; two (10.5\%) women cancelled and four $(21 \%)$ women failed to attend the clinic again. It is possible that the non-attenders had the IUS inserted elsewhere but this was not looked at in this study.

\section{Women choosing Implanon}

Following sterilisation counselling 13 women chose to have Implanon inserted and they were given appointments to return to the clinic to have this done. Nine $(69 \%)$ women attended and had Implanon inserted and four $(31 \%)$ women did not return for the procedure. It is possible that the nonattenders had Implanon inserted elsewhere but this was not followed up in this study.

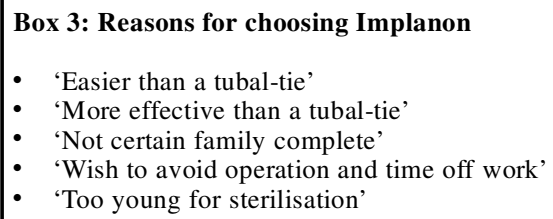

\section{Couples choosing vasectomy}

Six couples wished to be referred for vasectomy counselling and they were given appointments at the weekly vasectomy counselling clinic. Of these five couples attended for counselling and four went ahead with a vasectomy operation. The main reason for couples choosing a vasectomy was that it is more effective and easier to perform than a female sterilisation.

\section{Discussion}

Sterilisation is the most commonly used method of contraception worldwide. ${ }^{1}$ Despite this the number of female sterilisations performed in England and Wales is falling. Since 1998 the total number has fallen by $27 \%$ from 47288 in 1998 to 34784 in $2001 .^{9}$ Over this time the number of female sterilisations performed in the Newcastle area has also declined. In 1998351 laparoscopic sterilisations were performed at the RVI. This figure fell by $45 \%$ to 195 in 2001 . We feel that this reduction is due partly to the comprehensive counselling the women receive in the FPC and partly to the fact that other long-term, effective and reversible methods of contraception are now available. Women are no longer seeing sterilisation as the only reliable method of contraception available to them. Our study would suggest that female sterilisation is not always what women want. Only $61 \%$ of women went ahead with the procedure after counselling, and out of all the appointments offered for counselling only $41 \%$ underwent a laparoscopic sterilisation.

When women were asked why they had chosen the IUS or implant their replies centred around 'reversibility', 'avoidance of surgery' and 'the non-contraceptive benefits of these hormonal methods', particularly in reducing menstrual blood loss. The US Collaborative Review of Sterilization highlighted the numbers of women who regret making the decision to use a permanent method of contraception. The cumulative probability of expressing regret during follow-up interview within 14 years of female sterilisation was $20.3 \%$ for women aged 30 years or younger at the time of surgery. ${ }^{10}$ It was even higher in this age group if women were sterilised within 1 year after the birth of their youngest child (22.3\%). Moreover, the 5-year cumulative probability of hysterectomy was $8 \%$ among those previously sterilised compared to $2 \%$ if their partners 


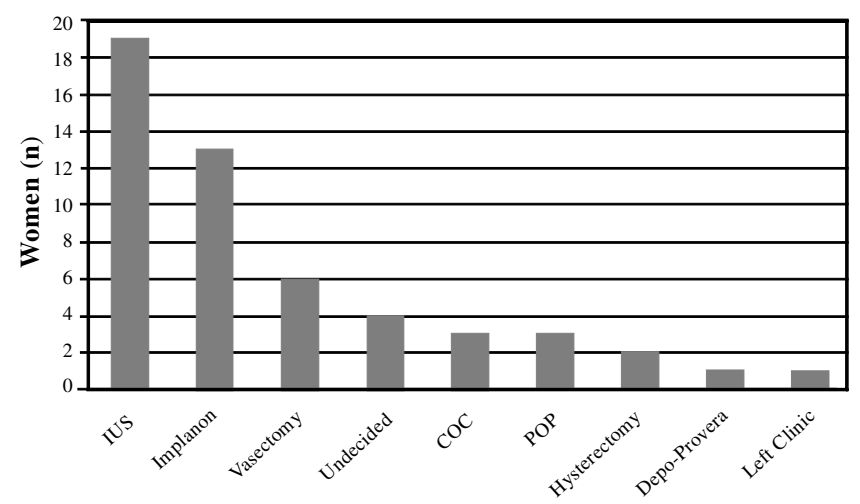

Alternative methods of contraception chosen

Figure 3 Alternative methods of contraception chosen $(n=52)$. COC combined oral contraceptive; IUS, intrauterine system; POP, progestogen-only pill.

had undergone a vasectomy. ${ }^{11}$ At 14 years post-sterilisation the cumulative probability of having a hysterectomy was $17 \%$, with rates as high as $35 \%$ and $46 \%$ in women with endometriosis or prolonged menses at the time of the original surgery. ${ }^{12}$ This leads us to conclude that health professionals working in primary and secondary care hold the key to improving women's health by providing careful and up-to-date information.

Our non-attendance rate during the study period was $32 \%$. Studies report figures ranging from $5 \%$ to $34 \%$ for non-attendance at outpatient clinics. ${ }^{13}$ The non-attendance rate is higher in deprived areas and the peak age range for hospital non-attenders is between 20 and 30 years. This is the age range of women commonly referred to our unit for sterilisation counselling so perhaps a high non-attendance rate is not surprising. However, we plan to try to reduce our non-attendance rate by altering the way we offer appointments for sterilisation counselling. Once we receive a GP referral for female sterilisation we will write to the woman asking her to telephone the clinic secretary to arrange her own appointment. Women will be able to choose a date and time convenient to themselves and if they have changed their mind about being sterilised they do not need to arrange an appointment at all.
We plan to re-audit the women who chose alternative methods of contraception in 12 and 24 months' time. We want to look at the continuation rate of the alternative methods and to discover whether any women have subsequently gone on to be sterilised after all.

\section{Acknowledgements}

The authors would like to thank Mrs J A Duffy, Mr M M Singh, Mrs C McGarry and the staff at the Family Planning Unit for their contribution to this project.

Statements on funding and competing interests

Funding. None identified.

Competing interests. None identified.

References

1 Rioux JE, Daris M. Female sterilisation: an update. Curr Opin Obstet Gynecol 2000; 13: 377-381.

2 Edozien L. Counselling for female sterilisation. Br J Fam Plann 1997; 23: 14-15.

3 Fortney JA, Feldblum PJ, Raymond EG. Intrauterine devices. The optimal long-term contraceptive method? J Reprod Med 1999; 44: 269-274.

4 Male and female sterilisation. London: Family Planning Association, 2000.

$5 \quad$ Male and female sterilisation. Evidence-based clinical guidelines No. 4. London: Royal College of Obstetricians and Gynaecologists, April 1999.

6 Minogue M. Providing good standards of care. Journal of the MDU 1999; 15: 21-22.

7 Peterson HB, Xia Z, Hughes JM, et al. The risk of pregnancy after tubal sterilisation: findings from the US Collaborative Review of Sterilisation. Am J Obstet Gynecol 1996; 174: 1161-1170.

8 Edwards J, Moore A. Implanon: a review of clinical studies. Br J Fam Plann 1999; 24(4)(Suppl.): S3-S16.

9 Hospital Episode Statistics: December 1998, December 2001. www.doh.gov.uk/hes/

10 Hillis SD, Marchbanks PA, Tylor LR, et al. Poststerilization regret: findings from the United States Collaborative Review of Sterilization. Obstet Gynecol 1999; 93: 889-895.

11 Hillis SD, Marchbanks PA, Tylor LR, et al. Higher hysterectomy risk for sterilized than nonsterilized women: findings from the United States Collaborative Review of Sterilisation. Obstet Gynecol 1998; 91: 241-246.

12 Hillis SD, Marchbanks PA, Tylor LR, et al. Tubal sterilization and long-term risk of hysterectomy: findings from the United States Collaborative Review of Sterilization. Obstet Gynecol 1997: 89; 609-614.

13 Sharp D, Hamilton W. Non-attendance at general practices and outpatient clinics. BMJ 2001; 323: 1081-1082.

\section{Mifepristone and misoprostol for medical termination of pregnancy: the effectiveness of a flexible regimen}

Ranjan Basu, MD, MRCOG, Senior SHO; Tina Gundlach, RGN, DPN NS, Sister; Margaret Tasker, FRCOG, MFFP, Consultant Obstetrician and Gynaecologist, Women's Health Care Clinic, Department of Obstetrics and Gynaecology, Royal Bolton Hospital, Bolton, UK

Correspondence: Dr M Tasker, Department of Obstetrics and Gynaecology, Royal Bolton Hospital, Minerva Road, Farnworth, Bolton BL4 OJR, UK. E-mail: Barbara.Davies@boltonh-tr.nwest.nhs.uk

(Accepted 23rd Decemberr 2002)

Journal of Family Planning and Reproductive Health Care 2003; 29(3): 139-141

\begin{abstract}
Background. Mifepristone, followed 48 hours later by administration of misoprostol, is a well-established regimen for medical termination of pregnancy (TOP). Although this regimen is effective, its inflexibility may limit its provision in an outpatient service.

Objective. To confirm that misoprostol administration is effective whether administered 24, 48 or 72 hours after oral mifepristone.

Design. Observational study of 234 consecutive women with pregnancies up to 83 days' gestational age in whom
\end{abstract}

medical TOP was performed during the period December 2000-July 2001.

Setting. Women's Health Care Department, Royal Bolton Hospital, Bolton, UK.

Results. There was a high success rate for complete abortion in all groups whether mifepristone was administered 24, 48 or 72 hours prior to misoprostol.

Conclusion. This study suggests that a more flexible regimen of mifepristone/misoprostol administration for medical TOP is effective in routine clinical practice. 